\title{
Application of Plane Formation to Landscape Design
}

\author{
Chuncai Zhang \\ Huanghe Science and Technology College \\ Zhengzhou, China \\ 1352101158@qq.com
}

\begin{abstract}
As one of the compulsory courses of landscape design, importance of plane formation in landscape design is evident. Along with development of landscape design, requirements for artistic quality of landscape from the society become gradually higher. Modern landscape design has already been impregnated with the art of formation. This paper put forward approaches and suggestions on how to use formation art for reference, and how to absorb formation art through study on application of plane formation to modern landscape design.
\end{abstract}

\section{Keywords—plane formation form; landscape design}

\section{INTRODUCTION}

As the fundamental subject for specialty of artistic design and the basic theory of modern visual communication art, plane formation builds images via profiles, combines different basic forms into patterns in the plane as per certain rules and discusses the constitution theory, rules and principles of beauty in form in all plane arts (Liang \& Li, 2009). Plane formation plays an important role in the modern artistic design, for the formal beauty like symmetry, balance and sequence that it conveys conforms to the aesthetic principle of the modern; besides, it has strong supporting points in traditional aesthetics. This paper mainly studies the method and rules for application of basic elements of plane formation and principles of beauty in form to landscape design for the purpose of better and more application of plane formation to the modern landscape design.

\section{APPLICATION OF BASIC ELEMENTS OF PLANE FORMATION TO LANDSCAPE DESIGN}

As basic elements of visual space, point, line and surface are basic language to represent visual image. Any design practice can be summarized as combination of point, line and surface. Similarly, basic elements of plane formation are point, line and surface. Point, line and surface in art are relative concepts, which are not as precise as mathematic concepts. Several points in the same line can convert to line, while lines arranged together can convert to surface, which can change into points when it is put in a bigger environment. They are interchangeable in specific environments.

\section{A. Application of points.}

Point, as one of the basic elements, plays a significant role in landscape design. The size and shape of points can be determined according to the size of space where it is and the relation between objects, and can be compared by sensation of people. Objects of any shape can be understood as points in specific environments. For example, trees, pavilions, stones and sculptures all can be understood as points. The point is easy to attract people's attention and becomes the visual center. In addition, point can enrich model and break drabness. It makes the finishing point in design. For example, a sculpture placed in the center of a plaza is used to break the spacious atmosphere, and to give prominence to the key points. Point can also divide space, for example, continuous alee trees can serve as a border; well-arranged stones can divide and beautify space.

Points have all kinds of shapes, regular and irregular. The smaller the point is, the stronger the impression it gives is, however, it is delicate. When the point becomes bigger gradually, the sensation it gives is more like surface. At this time, the shape of the point, geometric form or concretization form, plays an important role. However, in any case, the point, as a tiny feature, should try to adopt a simple, neat and powerful shape. In plane formation, points include large points, small points, square points and round points, which all have their own effective meaning. In practice of landscape design, we can gain rich spatial effects according to the psychological suggestion that people get from the visual characteristics of these points.

\section{B. Application of lines.}

As the essential condition among modeling elements to form visual image, the line is the basic single dimension graphic recording, the result of linear array of innumerable points. Lines have clearer direction-sense than points, so they can express development process of specific objects and thoughts. Modeling and composition of picture is impossible without lines (Zheng 2010). In the discipline of arts, line is the simplest and most effective modeling language for either painting or design. All kinds of lines are ever changing in modeling performance, and the visual center transfers along with changing of lines. Layout structure of landscape is composed of lines of different shapes, while the variety of form relies on changing of lines. Different linear designs can generate different visual effects. In landscape design, lines mainly consist of straight lines and curves, among which, the straight line is the most widely used linearity in landscape design, for straight lines give the sense of being strong, stout, stable, open, simple and bright. Straight lines are often used in formal gardens. In order to give prominence to the absolute regality of emperor, design of the ancient palace often has a long axle line, which is majestic. The curve gives the sense of 
being free, comfortable, fluent, brisk, elegant and romantic. It is easy to assort with the natural landscape, so it is often used in recreational and lively places.

\section{Application of surface.}

Surface is the space surrounded by lines. It is the widely used modeling element in landscape design. Main elements of surface are forms and areas. Forms like grassplot, lake surface and buildings that have certain areas can be called as surface. Surface can constitute all kinds of forms through spreading, stacking or intersecting, so its expressive force is abundant. Surface can be divided into geometric form surface and free form surface according to shapes. The geometric form surface is often used in landscape design of plaza, which is generated by combination and division of round and square surfaces, so as to create a spacious and regular environment. The free form surface is often used in landscape of gardens and amusement parks. Combined with the geometric form surface, it can create the effect of a winding path leading to a secluded place.

In practice of design, we can use pure points, lines and surfaces as the basic archetype to create the decorative form with special beauty through spatial variation of translating, rotating and twisting, and adding combination of materials of different senses of reality.

\section{APPLICATION OF THE PRINCIPLE OF BEAUTY IN FORM OF PLANE FORMATION TO LANDSCAPE DESIGN}

The principle of beauty in form refers to summarization of rules of beauty forms according to experiences gathered by human being during the process of creating beauty. In landscape design, we first create graphics by making use of basic elements of plane formation, and then handle the relations among elements as per principles of beauty in form to integrate them into the general planning of landscape design. Principles of beauty in form are mainly composed of contrast and unity, symmetry and balance, rhythm and cadence.

\section{A. Contrast and unity.}

Contrast is mainly embodied in difference of each element of design, which is widely used in landscape design. Aspects of contrast include contrasts of shapes, colors and primary and secondary. The purpose of contrast is to avoid monotonous scenes in design, and to enrich design forms, which is helpful to increase people's visual interest. Design of many parks takes the pavilion or sculpture as the primary landscape node, which contrasts other secondary landscape. In design of plants, "a single red flower in the midst of thick foliage" also reflects the technique of contrast. In landscape design, due to diversity of design elements, if the contrast is slight, the scene will be mild and dull; if the contrast is strong, the visual effect will be abrupt and inharmonious. Therefore, intensity of contrast must be well grasped when contrast technique is adopted so as to gain the best visual effect.

Unity refers to the harmonious relations among all elements that constitute the design. The principle of unity has influence on size, shape, color, and texture of every element of design in the environment. No matter what kind of principles of beauty in form we use in our design, it should be carried out in accordance with the major principle of unity. When use contrast in design, we should find commonness of all design elements. Try to seek variety and unity from variety. Only when our design is unified, can the modeling elements in it give the sense of "an integral whole".

\section{B. Symmetry and balance.}

Marxist aesthetics thinks that the symmetry principle of arts is the reflection of actual law of the objective world in arts rather than the arbitrary outcome of artists. Symmetry is the mechanism of existence of human being, animals, many plants and artificiality. It is a kind of form of beauty (Wang 2005). In landscape design, symmetry is often used together with axes. It has precise composition of picture when used for overall layout of landscape, so it is often used in royal gardens, plazas and memorial parks. It is indicative of serious and grand effects. In addition, symmetry can be used for local composition of pictures, at this time, local place will have evident axes or points of symmetry, such as symmetry of points, symmetry of lines and symmetry of surface. This is generally used at both sides of exits and entrances or plazas, for it gives the sense of safety and stabilization.

Balance refers to development of symmetry in form. Because the symmetrical layout in certain places will give the sense of stiffness, and balance can make up the simplification to make the space of gardens lively and full of variety, in classical gardens of China, the handling method of balance is usually adopted on roads and arrangement of plants in order to achieve the effect of being infinite in variety.

\section{Rhythm and cadence.}

Rhythm and cadence are music terms, but now, they are widely used in art form. It is one of the means of artistic expression to create beauty in form. Rhythm is the regular strong and weak, long and short phenomenon that appears alternatively in music. The cadence is lingering charm and rhythm of music or poetry. In design practice, rhythm and cadence mainly show the dynamic and continuous beauty that is regular, orderly and full of variety. It is generated from regular continuous repetition of certain elements of composition of a picture. In landscape design, rhythm and cadence are mainly embodied in arrangement of plants, landscape sketch, roads and pavement. For example, in arrangement of plants, plants along both sides of the road should have three-dimensional contour line and spatial variation. There should be high and short plants so as to create rhythm and cadence to prevent the layout from being stereotyped.

\section{CONCLUSION}

Influence of plane formation on modern landscape design is great. Besides, it serves as an important guide, especially for beginners, for it helps them to conceive sketch and develop their thinking. The principle of beauty in form of plane formation takes the whole situation into account during the process of design. It provides reference for us to improve visual aesthetic quality of design and embody modern flavor. 


\section{REFERENCES}

[1] Liang, J., Li, Y.H. "Application of Plane Formation Art in Design of Gardens." J. Journal of Shijiazhuang Vocational Technology Institute, (6): 52. (2009)

[2] Wang, L.Q., "Plane Formation". M. Chongqing: Chongqing University Press, 25(2005)

[3] Zheng, Y.L. "Study on Rules of Application of Plane Formation in Design of Modern Landscape". J. Journal of Heilongjiang Vocational Institute of Ecological Engineering, (1): 15. (2010). 